\title{
MOLECULAR BIOLOGICAL STUDIES OF ADULT AND METACERCARIAL STAGES OF PETASIGER EXAERETUS DIETZ, 1909 (DIGENEA: ECHINOSTOMATIDAE)
}

\author{
Gábor CECH*, Kálmán MOLNÁR and Csaba SZÉKELY \\ Institute for Veterinary Medical Research, Centre for Agricultural Research, \\ Hungarian Academy of Sciences - Fish Pathology and Parasitology, Hungária krt. 21, \\ H-1143 Budapest, Hungary
}

(Received 9 December 2016; accepted 4 April 2017)

\begin{abstract}
Molnár et al. (2015) reported two types of echinostomatid metacercariae in the lateral line organ of Hungarian fish species. Type 1 metacercariae possessed 27 collar spines and 16 uniform and three larger dorsal spines, whereas Type 2 metacercariae bore 27 collar spines and 19 equal-sized dorsal spines. In the recent work, molecular studies carried out on the ITS region and partial 28S rDNA sequences of two types of echinostomatid metacercariae and the sequences of adult stages of the species of Petasiger Dietz, 1909 collected from cormorants (Phalacrocorax carbo L.) showed that some of the Type 2 metacercariae corresponded to Petasiger exaeretus Dietz, 1909, whereas other morphologically similar metacercariae were identified as Petasiger phalacrocoracis (Yamaguti, 1939). The sequences of the Type 1 metacercariae with three larger dorsal spines could not be identified with any of the known sequences from echinostomatid trematodes.
\end{abstract}

Key words: Digenea, Petasiger, ITS, $28 \mathrm{~S}$ rDNA, metacercaria

In a recent paper, Molnár et al. (2015) reported finding echinostomatid metacercariae in the lateral line organ of some Hungarian fish species. These metacercariae, characterised by 27 collar spines, could be related to Paryphostomum Dietz, 1909 and Petasiger Dietz, 1909 species infecting the intestine of fish-eating birds, especially cormorants. Two types of metacercariae were collected. One of them (Type 1) had 16 uniform and three larger dorsal spines, whereas the other (Type 2) had 19 similarly sized dorsal spines. Later, by dissecting the gut of cormorants [Phalacrocorax carbo (Linnaeus)], the same authors collected two echinostomatid species, Petasiger phalacrocoracis (Yamaguti, 1939) and Paryphostomum radiatum (Dujardin, 1845). By analysing the ITS sequences (including parts of 18S rDNA, ITS1, 5.8S rDNA, ITS2 and parts of $28 \mathrm{~S}$ rDNA) of the two metacercarial types and adult specimens of Paryphostomum radiatum and Petasiger phalacrocoracis, the authors concluded that

*Corresponding author; E-mail: cech.gabor@agrar.mta.hu; Phone: 0036 (1) 467-4079; Fax: 0036 (1) 467-4076 
the sequences of five samples of Type 2 metacercariae corresponded to those of adult $P$. phalacrocoracis and to the sequence of $P$. phalacrocoracis AY245709 deposited in GenBank. The sequences of Type 1 metacercariae differed significantly from those of $P$. phalacrocoracis and any other genetically characterised species. A sixth sequence of one of the Type 2 metacercariae (PA3) gave a surprising result and, consequently, the authors postponed its study.

In recent years, two studies using molecular markers have been published on species of the genus Petasiger. Georgieva et al. (2012) described the life cycle of Petasiger islandicus Kostadinova et Skirnisson, 2007 applying 28S rDNA and nadI sequences, and Selbach et al. (2014) described four new cercariae of Petasiger spp. based on their morphology and $28 \mathrm{~S}$ rDNA and nadI sequences. Recently, Tkach et al. (2016) have provided a comprehensive molecular phylogeny of the Echinostomatoidea Looss, 1899, using 28S rDNA sequences, which included Petasiger spp.

The present paper has a dual purpose. On the one hand, we report results showing that one sequence of a Type 2 metacercaria showed $100 \%$ identity with the sequences of adult Petasiger exaeretus Dietz, 1909. On the other hand, our studies were extended to analyse the 28S rDNA of Petasiger and Paryphostomum spp., proving that the sequences of the species studied by us differ from those of the Petasiger spp. published by both Georgieva et al. (2012) and Selbach et al. (2014).

\section{Materials and methods}

The collection of parasites, as well as the morphological and histological methods used basically corresponded to those described by Molnár et al. (2015). In that study, only specimens of Petasiger phalacrocoracis were recorded from the gut of cormorants. During a re-examination of the Petasiger collection, 14 specimens of Pet. exaeretus were also found in one cormorant among Pet. phalacrocoracis specimens. Two of these (KM2, KM4) were studied for their ITS (including part of the 18S rDNA, ITS1, 5.8S rDNA, ITS2 and part of the 28S rDNA) and partial 28S rDNA sequences. Similarly, the postponed sequencing of a Type 2 echinostomatid metacercaria (PA3) collected from a cyprinid fish, the roach [Rutilus rutilus (Linnaeus)], was carried out for both genes. Additionally, the 28S rDNA was partially sequenced for the Petasiger and Paryphostomum samples published by Molnár et al. (2015). Adult worms were identified according to the keys of Kostadinova (2005), Kostadinova et al. (2002) and Faltýnková et al. (2008).

\section{Molecular methods}

For DNA extraction, samples preserved in ethanol were centrifuged at $8,000 \times g$ for $5 \mathrm{~min}$, after which the ethanol was removed. The DNA was ex- 
tracted using a QIAGEN DNeasy ${ }^{\mathrm{TM}}$ tissue kit (animal tissue protocol; Qiagen, Hilden, Germany) and eluted in $100 \mu \mathrm{l}$ AE buffer. The ITS region (part of the $18 \mathrm{~S}$ rDNA, ITS1, 5.8S rDNA, ITS2 and part of the 28S rDNA) was amplified and sequenced as described by Molnár et al. (2015). Partial 28S rDNA was amplified using primers ZX-1 (forward; 50-ACC CGC TGA ATT TAA GCA TAT30) (Bray et al., 2009) and 1500R (reverse; 50-GCT ATC CTG AGG GAA ACT TCG-30) (Tkach et al., 2003) and following the thermocycling conditions described by Selbach et al. (2014). PCR fragments of 28S rDNA were sequenced directly for both strands using the PCR primers.

The sequence fragments were assembled using MEGA 6.06 (Tamura et al., 2013) and ambiguous bases clarified using corresponding ABI chromatograms. Nucleotide sequences of the ITS region and 28S rDNA were aligned with the software CLUSTAL W (Thompson et al., 1994). The alignments were corrected manually using the alignment editor of the software MEGA 6.06. DNA pairwise distances were calculated with the MEGA 6.06 software using the Tamura-Nei substitution model. Maximum likelihood (ML) and Bayesian inference (BI) analyses were performed. The liver fluke (Fasciola hepatica Linnaeus, 1758) was chosen as the outgroup. The dataset was tested using MEGA 6.06 for the nucleotide substitution model of best fit and the model shown by the Akaike Information Criterion (AIC) was chosen. ML analyses of the ITS region and 28S rDNA were performed under the GTR $+\mathrm{G}+\mathrm{I}$ model. Bootstrap values based on 1000 resampled datasets were generated. BI was computed by Topali 2.5 (Milne et al., 2004). The likelihood parameters for BI were based on the GTR + G +I model. Posterior probabilities (PP) were estimated over 1,000,000 generations via two independent runs of four simultaneous MCMCMC chains with every 100 th tree saved. The first $25 \%$ of the sampled trees were discarded as 'burn in'. The ML trees were visualised using the tree explorer of MEGA 6.06.

\section{Results}

The metacercariae were recovered from an extensive infection of roach, as described previously (Molnár et al., 2015). The infection mainly affected the scales of the lateral line organ. Two types of metacercariae were differentiated.

The single metacercaria sample (PA3), which gave a surprising molecular result, belonged to the second type of metacercariae described by Molnár et al. (2015). That type of metacercariae had a cyst size of $128-157 \times 105-115 \mu \mathrm{m}$ and bore 27 collar spines: four pairs of angle spines of $28-40 \mu \mathrm{m}$ and 19 dorsal spines all of a similar size $(17-23 \mu \mathrm{m})$ (Fig. 1). The excysted metacercaria of sample (PA3) had a size of $425 \times 115 \mu \mathrm{m}$ (Fig. 2). Morphologically it was completely identical with the metacercariae of Petasiger phalacrocoracis (PA1, PA2, PA4, CK1, CK2). 


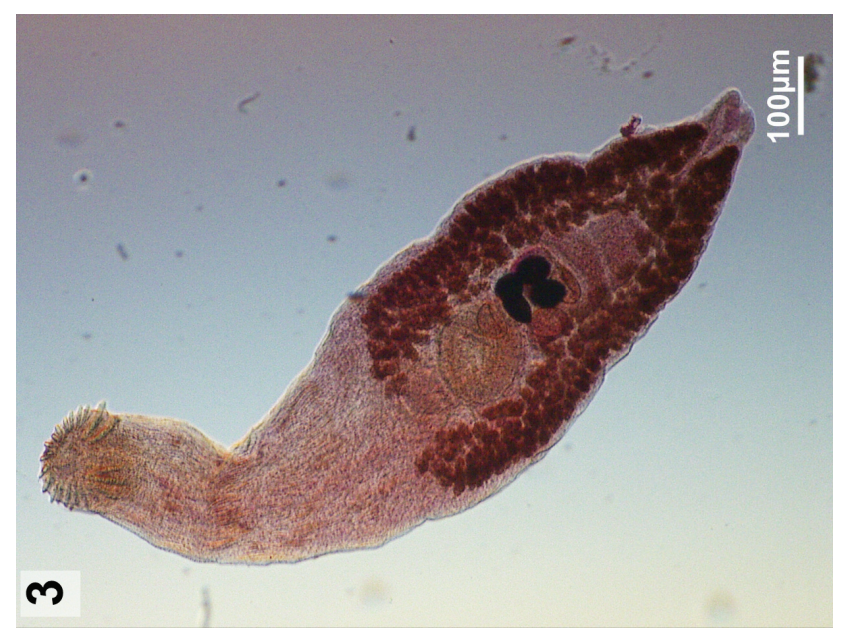

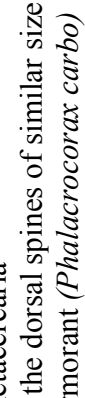

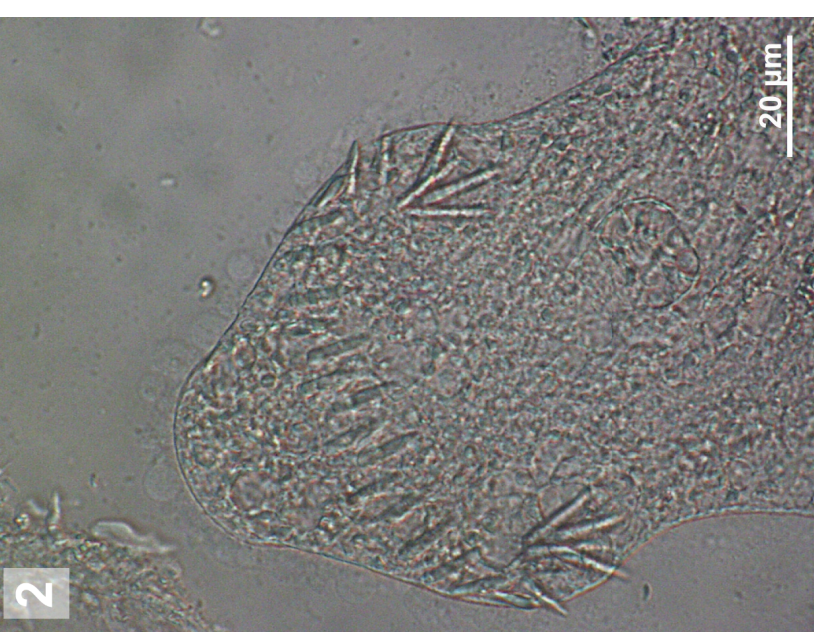

焉

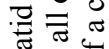

$\because$ bo

总言层

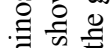

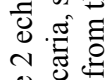

군

$F$

浣

政

氙

क

응

ते है

次

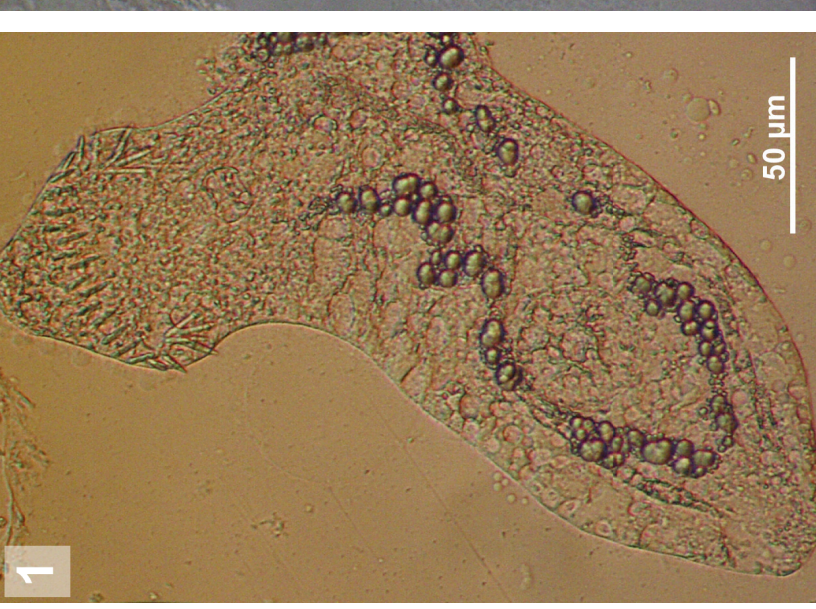

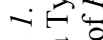

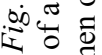

s.

:

章 冡

$i \dot{r}$

这这 


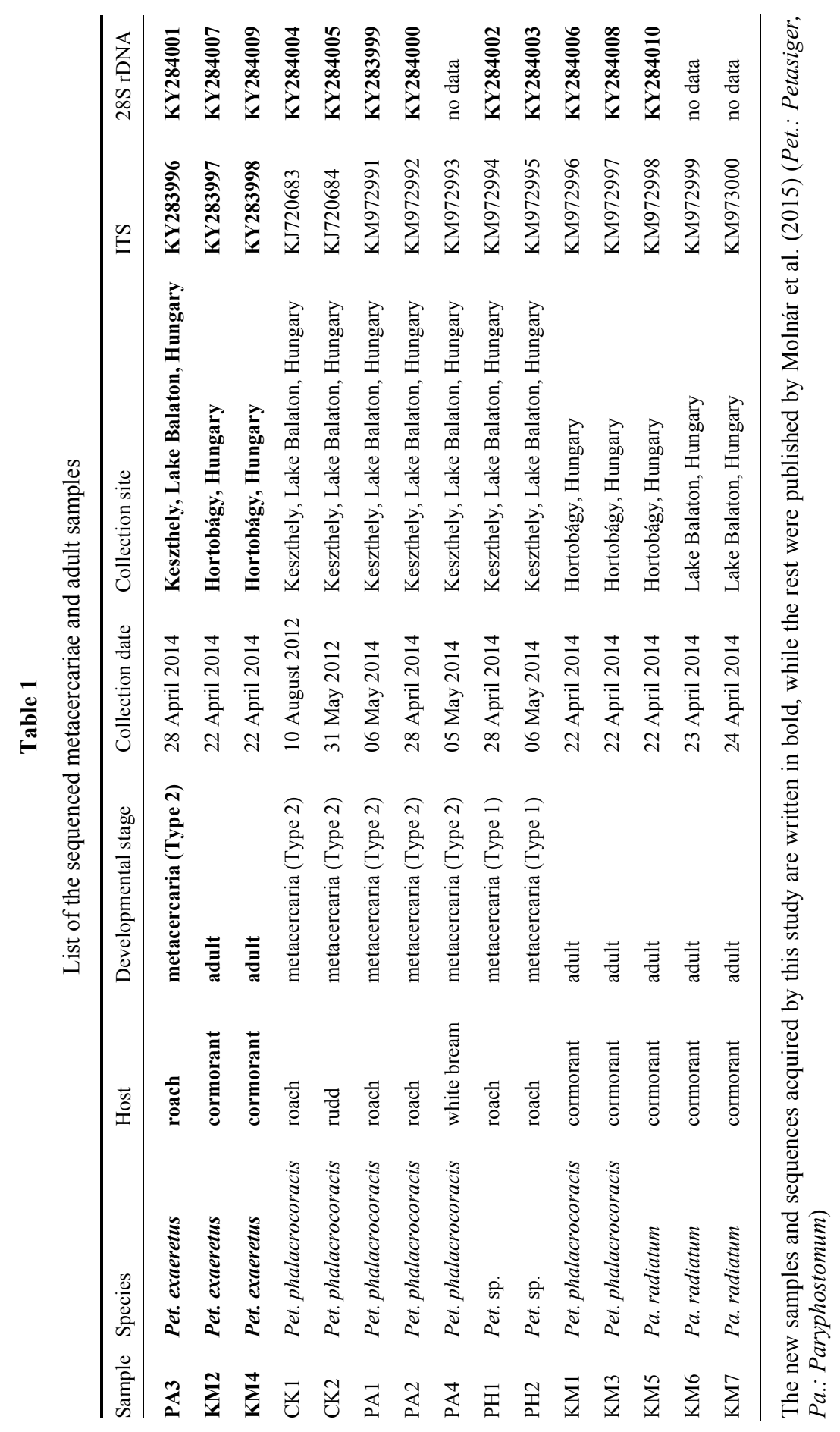


During an additional study of 12 Phalacrocorax carbo specimens caught during 2016, further echinostomatid trematodes were collected from the gut (Fig. 3). At this time, in addition to the previously reported species Paryphostomum radiatum and Pet. phalacrocoracis, the presence of a third species, Pet. exaeretus, was confirmed in these cormorants.

The ITS region and the 28S rDNA were sequenced for samples PA3, KM2 and $\mathrm{KM} 4$, in addition to the $28 \mathrm{~S}$ rDNA for the samples previously reported by Molnár et al. (2015) (Table 1). The sequences of the ITS region were more than $1,300 \mathrm{bps}$ long and the final alignment, containing the examined sequences and those downloaded from GenBank, was 1,088 bps long. Partial sequences of the 28S rDNA were about 1,230 bps long, with the corresponding alignment being 1,231 bps long. Phylogenetic analysis showed that the ITS region and the partial 28S rDNA region of metacercariae and adult stages of Petasiger and Paryphostomum spp. corresponded (Figs 4A and 4B) and proved that the sequences of one of the Type 2 metacercariae (PA3) were virtually identical with those of adult specimens of Petasiger exaeretus (KM2, KM4) isolated from the gut of cormorants. The samples were topologically separated from other Petasiger or Paryphostomum $(\mathrm{Pa}$.) species with high bootstrap. The ITS sequences of the three Pet. exaeretus sequences showed remarkable differences from the species Pet. phalacrocoracis (7.8-7.9\%), Pa. radiatum (7.4-7.5\%) and the as yet unidentified Type 1 metacercariae (8.2-8.3\%). The partial 28S rDNA sequences gave similar results, i.e. the Pet. exaeretus samples collected by us were identical with the specimen deposited in GenBank (KT956923) and differed from Pet. phalacrocoracis by $1.6-1.7 \%$, from $P a$. radiatum by $1.6 \%$ and from the Type 1 metacercaria by $1.6 \%$. Pet. phalacrocoracis and $P a$. radiatum samples were identical or nearly identical $(0.0 \%-0.3 \%$ differences $)$ with the deposited sequences (JQ425593, KT956926, KTKT956927). The sequences of the Type 1 metacercariae did not match those of any other genetically characterised species. The partial 28S rDNA sequences of all samples studied by us exhibited a relatively great difference from the sequences of Pet. islandicus Kostadinova \& Skírnisson, 2007 (3.1\%) and the cercariae of Petasiger spp. (3-4\%) described by Selbach et al. (2014).

\section{Discussion}

The ITS and 28S rDNA sequences of adult specimens of Petasiger exaeretus and a single metacercaria (PA3) yielded an unexpected result. Previously, we had supposed that the sequences of Pet. exaeretus might correspond to those of the Type 1 metacercariae with uneven dorsal spines. Instead of this, a metacercaria (PA3) which corresponded morphologically to the metacercariae of Pet. phalacrocoracis proved to be identical with the adult specimens of $P$. exaeretus, whereas the Type 1 metacercaria $(\mathrm{PH} 1, \mathrm{PH} 2)$ remained unidentified. There are other 


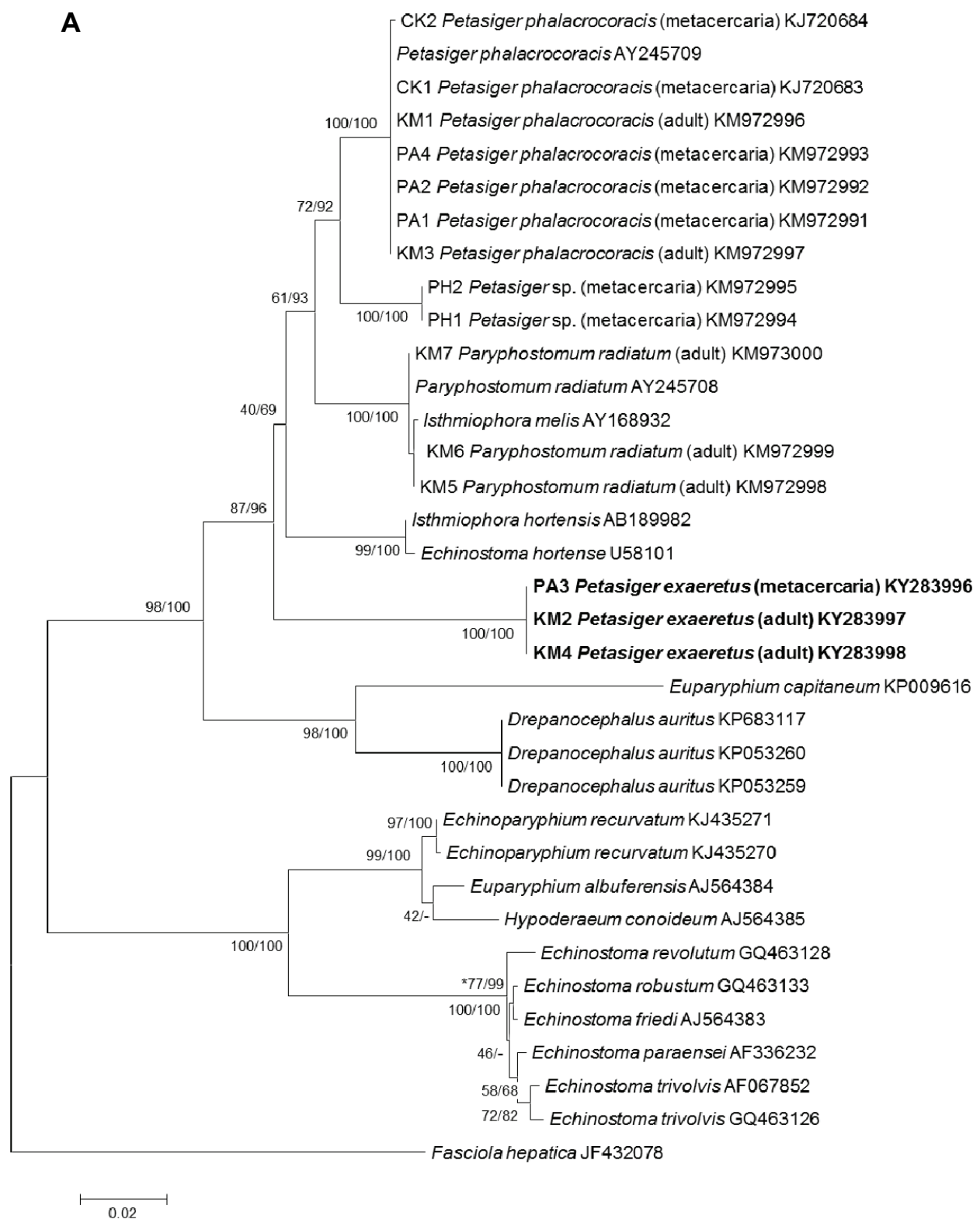




\section{B}

PA1 Petasiger phalacrocoracis (metacercaria) KY283999

CK1 Petasiger phalacrocoracis (metacercaria) KY284004

PA2 Petasiger phalacrocoracis (metacercaria) KY284000

99/95 Petasiger phalacrocoracis KT956926

KM3 Petasiger phalacrocoracis (adult) KY284008

9/74 KM1 Petasigerphalacrocoracis (adult) KY284006

0:74 36- Petasiger phalacrocoracis JQ425593

CK2 Petasiger phalacrocoracis (metacercaria) KY284005

44/-

99/100 PH1 Petasiger sp. (metacercaria) KY284002

PH2 Petasiger sp. (metacercaria) KY284003

3/56 K KM5 Paryphostomum radiatum (adult) KY284010

98100 Petasiger radiatus KT956927

$96 / 71$

Isthmiophora melis KT359583

100/100- Euparyphium melis AF 151941

Petasiger exaeretus KT956923

PA3 Petasiger exaeretus (metacercaria) KY284001

100/100 KM2 Petasiger exaeretus (adult) KY284007

KM4 Petasiger exaeretus (adult) KY284009

100 Drepanocephalus spathans JN993269

7376 Drepanocephalus auritus KP683117

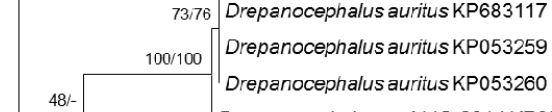

\begin{tabular}{|l|l}
\hline Drepanocephalus auritus KP053260 \\
Drepanocephalus sp. MJG-2014 KP053261
\end{tabular}

$\bigsqcup_{74 / 57}$ Chaunocephalus ferox KT447522 Euparyphium capitaneum KP009618

99/100 Petasiger sp. 1 AK-2014 KM191801

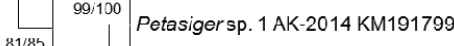

81/85 Petasigersp. 1 AK-2014 KM191800

47/87_ Petasigersp. 2 AK-2014 KM191802

97:100 [- Petasiger isiandicus JQ425592

Petasigersp. 4 AK-2014 KM191807

43/66 Petasigersp. 3 AK-2014 KM191803

Petasigersp. 3 AK-2014 KM191806

99/100 Petasigersp. 3 AK-2014 KM191805

Petasigersp. 3 AK-2014 KM191804

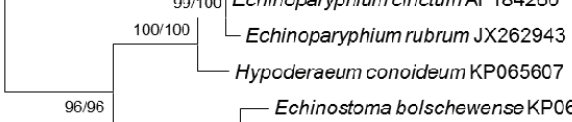

$96 / 9$

paraulum KP065604

87/91 L Echinostoma paraenseiEU025867

Fasciolahepatica EU025874

Fig. 4. Maximum likelihood tree (A: ITS; B: $28 \mathrm{~S}$ rDNA) of the commonly found echinostomatid metacercariae from the lateral line scales of roach and adult worms from cormorants in relation to echinostomatid material deposited in GenBank. Posterior probabilities for Bayesian inference (BI) are given behind the bootstrap values for ML (hyphen means unsupported by BI). New samples (PA3, KM2, KM4) and newly acquired 28S rDNA sequences of the previously published samples (Molnár et al., 2015) are in bold 
Petasiger species described from Europe (Kostadinova, 2005) for which sequence data are not yet available; these include Pet. grandivesicularis Ishii, 1935, Pet. megacanthus (Kotlán, 1922) and Pet. pungens (Linstow, 1893). A fifth species, Pet. neocomense Fuhrmann, 1927, has only nadI sequences in GenBank and, consequently, could not be compared with our samples. The abovementioned five species have 19 collar spines rather than 27 , and it is unlikely, therefore, that the Type 1 metacercaria belongs to any of these. Those species with 19 collar spines were elevated to full generic rank, as Neopetasiger Baschkirova, 1941, by Tkach et al. (2016), which would represent the morphological and molecular separateness of these species. The cercaria samples isolated by Selbach et al. (2014) also had 19 collar spines, so it is expected that the $28 \mathrm{~S}$ rDNA sequences of Pet. exaeretus, Pet. phalacrocoracis, Pa. radiatum and the Type 1 metacercariae will not match any of them. Tkach et al. (2016) also proposed that $P a$. radiatum should be transferred from the genus Paryphostomum to Petasiger. We agree with the opinion of Selbach et al. (2014) that there could well be a much higher diversity of Petasiger species based on the number of described cercariae as compared with adult forms (nine $v s$. five). Selbach et al. (2014) only discussed species with 19 collar spines, but the same diversity can be assumed for species having 27 collar spines. The Type 1 metacercaria cannot yet be regarded as a developmental stage of any of the genetically characterised species, as the nucleotide sequences of two genes contradict this; therefore, it likely indicates the presence of an as yet unknown species of Petasiger. Further studies on the Petasiger spp. of birds other than cormorants might help explain this result.

\section{Acknowledgements}

This study was supported by a grant (PD108813) from the Hungarian Scientific Research Fund (OTKA), the Bolyai Scholarship (BO/00417/15/4) and the GINOP-2.3.215-2016-00004 project: 'Establishing the sustainable angling-aimed management of Lake Balaton'. We would like to thank Lajos Juhász and Norbert Tóth (Department of Nature Conservation, Zoology and Game Management, Faculty of Agricultural and Food Sciences and Environmental Management, University of Debrecen, Debrecen, Hungary) for providing the cormorant samples.

\section{References}

Bray, R. A., Waeschenbach, A., Cribb, T. H., Weedall, G. D., Dyal, P. and Littlewood, D. T. J. (2009): The phylogeny of the Lepocreadioidea (Platyhelminthes, Digenea) inferred from nuclear and mitochondrial genes: Implications for their systematics and evolution. Acta Parasitol. 54, 310-329.

Faltýnková, A., Gibson, D. I. and Kostadinova, A. (2008): A revision of Petasiger Dietz, 1909 (Digenea: Echinostomatidae) and a key to its species. Syst. Parasitol. 71, 1-40. 
Georgieva, S., Kostadinova, A. and Skirnisson, K. (2012): The life cycle of Petasiger islandicus Kostadinova \& Skirnisson, 2007 (Digenea: Echinostomatidae) elucidated with the aid of molecular data. Syst. Parasitol. 82, 177-183.

Kostadinova, A. (2005): Family Echinostomatidae. In: Jones, A., Bray, R. A. and Gibson, D. I. (eds) Key to the Trematoda (Vol. 2). CAB International, Wallingford and The Natural History Museum, London, UK. pp. 9-64.

Kostadinova, A., Vaucher, C. and Gibson, D. I. (2002): Redescriptions of two echinostomes from birds in Paraguay, with comments on Drepanocephalus Dietz, 1909 and Paryphostomum Dietz, 1909 (Digenea: Echinostomatidae). Syst. Parasitol. 53, 147-158.

Milne, I., Wright, F., Rowe, G., Marshal, D. F., Husmeier, D. and McGuire, G. (2004): TOPALi: software for automatic identification of recombinant sequences within DNA multiple alignments. Bioinformatics 20, 1806-1807.

Molnár, K., Gibson, D. I., Cech, G., Papp, M., Deák-Paulus, P., Juhász, L., Tóth, N. and Székely, C. (2015): The occurrence of Petasiger metacercariae (Digenea) in an unusual site, within the lateral line scales of cyprinid fishes. Folia Parasitol. 62, Paper 017. 7 pp.

Selbach, C., Soldánová, M., Georgieva, S., Kostadinova, A., Kalbe, M. and Sures, B. (2014): Morphological and molecular data for larval stages of four species of Petasiger Dietz, 1909 (Digenea: Echinostomatidae) with an updated key to the known cercariae from the Palaearctic. Syst. Parasitol. 89, 153-166.

Tamura, K., Stecher, G., Peterson, D., Filipski, A. and Kumar, S. (2013): MEGA6: Molecular Evolutionary Genetics Analysis, version 6.0. Mol. Biol. Evol. 30, 2725-2729.

Thompson, J. D., Higgins, D. G. and Gibson, T. J. (1994): CLUSTALW: improving the sensitivity of progressive multiple sequence alignment through sequence weighting, position-specific gap penalties and weight matrix choice. Nucl. Acids Res. 22, 4673-4680.

Tkach, V. V., Kudlai, O. and Kostadinova, A. (2016): Molecular phylogeny and systematics of the Echinostomatoidea Looss, 1899 (Platyhelminthes: Digenea). Int. J. Parasitol. 46, 171-185.

Tkach, V. V., Littlewood, D. T. J., Olson, P. D., Kinsella, J. M. and Swiderski, Z. (2003): Molecular phylogenetic analysis of the Microphalloidea Ward, 1901 (Trematoda: Digenea). Syst. Parasitol. 56, 1-15. 